\title{
Tunneling magnetothermopower in magnetic tunnel junctions
}

\author{
Carlos López-Monís, Alex Matos-Abiague, and Jaroslav Fabian \\ Institute for Theoretical Physics, University of Regensburg, 93040 Regensburg, Germany
}

(Received 29 October 2013; published 18 February 2014)

\begin{abstract}
Thermally induced spin-dependent transport across magnetic tunnel junctions (MTJs) is theoretically investigated. We analyze the thermal analog of Slonczewski's model (as well as its limiting case-Julliere's model) of tunneling magnetoresistance and obtain analytical expressions for the junction thermopower and the tunneling magnetothermopower (TMT). The analytical model is tested numerically for the special case of an $\mathrm{Al}_{2} \mathrm{O}_{3}$-based MTJ, for which we analyze the dependence of the thermopower and TMT on the relative magnetization orientations, as well as on the barrier height and thickness. We show that a certain barrier height TMT vanishes, separating the region of positive and negative TMT. As its electrical prototype, this thermal spin transport model should serve as a phenomenological benchmark for analyzing experimental and first-principles calculations of thermopower in magnetic tunnel junctions. The analytical expressions can be used as a first estimate of the magnetothermopower of the junctions using $a b$ initio band structure data of the junction ferromagnets.
\end{abstract}

DOI: 10.1103/PhysRevB.89.054419

PACS number(s): 75.76.+j, 85.75.-d, 85.30.Mn, 72.20.Pa

\section{INTRODUCTION}

Traditionally, electric fields have been used as the main force to induce and explore spin-dependent transport in solidstate systems. This has lead to the fundamental and technologically profitable field of spintronics [1]. Nevertheless, in recent years we have witnessed how research on thermally driven spin-dependent transport has flourished, bringing forth a new field known as spin caloritronics, which merges spintronics with classical thermoelectricity [2]. The former deals with the interplay between the charge and spin degrees of freedom of carriers, while the latter with the generation of voltages induced by temperature gradients (and vice versa) [3]. Therefore, spin caloritronics addresses the interaction between spins and heat curents on the transport properties of a system. Although nominally it is a new field, already in the 1980s Johnson and Silbsee performed thermodynamic studies on spin-injection across ferromagnetic-nonmagnetic interfaces [4,5]. More recently, Gravier et al. measured spin-dependent heat transport across $\mathrm{Co} / \mathrm{Cu}$ multilayers [6,7]. However, the field came to prominence with the discovery of the so called spin-Seebeck effect [8-12].

In this framework, spin-valves have proved to be excellent systems for probing spin caloritronic phenomena [13-24]. An ordinary spin-valve is a heterostructure composed of two ferromagnetic materials separated by a mesoscopic nonmagnetic layer. Varying the relative orientation of the magnetizations of the ferromagnets allows us to study the spin-dependent properties of the system. When the nonmagnetic material is an insulator or a semiconductor, spin-valves are usually referred to as a magnetic tunnel junctions (MTJs). In order to explore the spin-dependent thermoelectric properties of a spin-valve, a temperature gradient is applied across the system (see Fig. 1), which induces charge, spin, and/or heat currents - or voltages.

In classical thermoelectricity, a material is characterized by its thermal conductivity, Peltier coefficient and thermopower. In the following, we shall focus exclusively on the latter property. The thermopower-also known as Seebeck coefficientmeasures the magnitude of an induced thermoelectric voltage in response to a temperature gradient across the material. When the spin degree of freedom is taken into account, in addition to the charge voltage, a spin accumulation might also be induced in the system. Analogously, the spin-Seebeck coefficient measures the magnitude of a spin accumulation induced by the temperature gradient [8-12]. Furthermore, when dealing with spin-valves, it is commonly studied the dependence of the thermopower on the relative magnetization orientations, which has been dubbed the magneto-Seebeck effect [14,21]. Similar to the tunneling magnetoresistance (TMR), a tunneling magnetothermopower (TMT) can also be defined [14].

Experimentally, Seebeck spin tunneling has been observed in ferromagnet/insulator/silicon tunnel junctions [13], realizing thermal spin-injection into semiconductors. The magnetoSeebeck effect was measured in MgO-based MTJs under heat gradients created optically [14], electrically [15,25], and even without an external heating source by using the heat dissipation of the tunneling current [16]. Giant thermoelectric effects have been observed in $\mathrm{Al}_{2} \mathrm{O}_{3}$-based MTJs [17]. Besides MTJs, all metallic spin-valves have also been probed for spin-dependent thermal properties [18].

From the theoretical point of view, spin-dependent thermal transport has been studied in ferromagnet/insulator/ferrmagnet MTJs [19]. In particular, a giant magnetothermopower effect has been predicted by magnon-assisted thermal transport [20]. Ab initio calculations have been performed for $\mathrm{MgO}$ based MTJs, where the TMT dependence on temperature was computed [21,26,27], and which has been used to understand previously mentioned experiments [14]. Similar calculations have been performed for GaAs-based MTJs [28]. Also, inspired by an aforementioned experiment [13], theoretical descriptions of thermal spin transport for ferromagnet/insulator/semiconductor MTJs have also been developed $[22,29]$. Finally, theory regarding all-metallic junctions has as well been recently studied [23,24,30,31].

Despite all this theoretical effort, to our knowledge, a more elaborated analytical description of the magneto-Seebeck effect in MTJs is still lacking. ${ }^{1}$ On the one hand, Slonczewski

\footnotetext{
${ }^{1}$ Except in the case of W. Lin et al. [17], where they used Julliere's model [38] to provide some insight to their experimental data.
} 


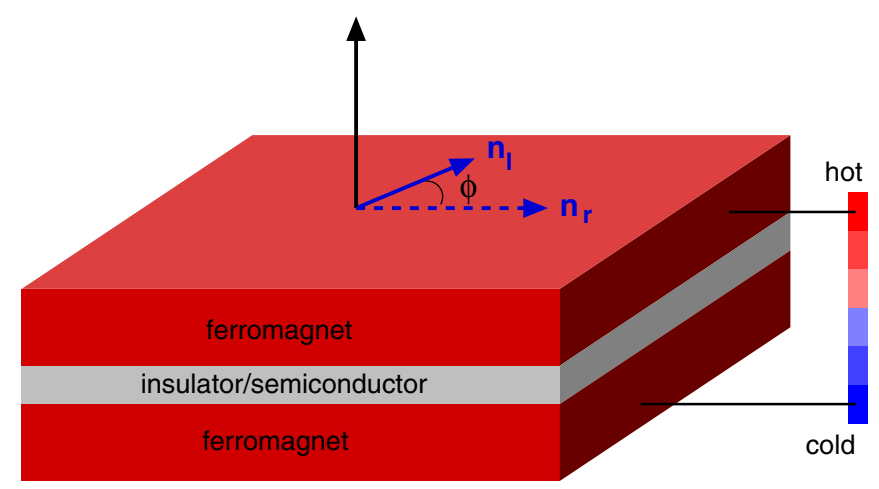

FIG. 1. (Color online) Scheme of a three-layer magnetic tunnel junction. A thermally induced current tunnels across the insulator/semiconductor tunneling barrier from one ferromagnet electrode into the other. The thermopower and TMT depend on the relative orientation of the magnetization of the left electrode $\mathbf{n}_{l}$ (solid arrow) with respect to the magnetization of the right electrode $\mathbf{n}_{r}$ (dashed arrow).

[32] developed an analytical model for describing the TMR in ferromagnet/insulator/ferromagnet MTJs. On the other, Mott derived a relation between the thermopower and the energy derivative of the conductance for low temperatures [33,34]. In this paper, we derive analytical expressions for both the thermopower and the TMT by combining Slonczewski's model with Mott's relation. We also study the limiting caseJulliere's model, which provides robust albeit highly simplified expression for the junction magnetothermopower. We believe the formulas obtained here should support both ab initio calculations and experiments.

The paper is organized as follows: the definitions of the thermopower and the TMT are given in Sec. II A, and the transmission probability of the MTJ is computed in Sec. II B. In Sec. IIC we derive the analytical formulas for the thermopower and the TMT. These analytical formulas are evaluated in Sec. III, where they are compared to the corresponding numerical calculations performed for the case of an $\mathrm{Al}_{2} \mathrm{O}_{3}$-based MTJ. Finally, a summary is given in Sec. IV.

\section{THEORY}

\section{A. Tunneling magnetothermopower}

In general, the current $I$ across a MTJ induced by a thermal gradient is given by [35]

$$
I=-G S \Delta T,
$$

where $G$ is the conductance, $S$ the thermopower or Seebeck coefficient, and $\Delta T$ the temperature difference between the electrodes. This tunneling current can be computed through [36]

$$
I=\frac{1}{e} \int g(E)\left[f_{L}(E)-f_{R}(E)\right] d E,
$$

where $f_{L}(E)$ and $f_{R}(E)$ are the Fermi-Dirac distributions of the left and right electrodes respectively,

$$
g(E)=\frac{e^{2}}{h} \frac{1}{(2 \pi)^{2}} \int T\left(E, \mathbf{k}_{\|}\right) d^{2} \mathbf{k}_{\|},
$$

and $T\left(E, \mathbf{k}_{\|}\right)$is the transmission probability associated with an electron with energy $E$ and transverse $k$ vector $\mathbf{k}_{\|}$.

Therefore, combining Eqs. (1) and (2) in linear response regime, the thermopower $S$ and the conductance $G$ are given by the following integrals:

$$
\begin{aligned}
S & =-\frac{1}{G} \int g(E)\left(-\frac{\partial f_{0}}{\partial E}\right)\left(\frac{E-\mu_{0}}{e T_{0}}\right) d E, \\
G & =\int g(E)\left(-\frac{\partial f_{0}}{\partial E}\right) d E,
\end{aligned}
$$

respectively, where $\mu_{0}$ and $T_{0}$ are the chemical potential and the temperature of the electrodes in equilibrium, respectively. Performing the change of variable $\epsilon=E-\mu_{0}$ in the integral in Eq. (4a), it is straightforward to show that, for the thermopower $S$ to be finite, $g(\epsilon)$ must not be an even function, i.e., $g(\epsilon) \neq g(-\epsilon)$.

The tunneling magnetothermopower, which measures the dependence of the thermopower $S$ on the relative in-plane magnetization orientations, is defined as

$$
\operatorname{TMT}(\phi)=\frac{S(0)-S(\phi)}{S(\phi)},
$$

where $\phi$ is the angle spanned between the magnetization vectors of the ferromagnetic layers (see Fig. 1).

\section{B. Computation of the transmission probability}

In order to study the thermopower, the transmission probability must be computed. For this purpose we use Slonczewski's model [32,36], which describes the ferromagnetic electrodes using the Stoner model [37], and the tunneling barrier by means of a rectangular potential. This model also assumes that the energy and transverse modes of the electrons are conserved. The advantage that this description has is that it allows to compute analytically the transmission probability of the MTJ.

Thereupon, the Hamiltonian we use for describing the MTJ is

$$
\mathcal{H}=\left(-\frac{\hbar^{2} \nabla^{2}}{2 m_{i}^{*}}+V_{i}\right) \mathcal{I}-\frac{\Delta_{i}}{2} \mathbf{n}_{i} \cdot \boldsymbol{\sigma},
$$

where the subscript $i$ describes the left $(l)$, central $(c)$, and right $(r)$ layers. The first term in Eq. (6) corresponds to the kinetic energy operator, where $m_{i}^{*}$ is the electron effective mass in the $i$ th layer. The second term is the rectangular potential barrier with $V_{l}=V_{r}=0$. The third term accounts for coupling between the magnetization and the electron spin in the ferromagnetic leads (Stoner model), where $\Delta_{i}$ and $\mathbf{n}_{i}$ correspond to the exchange energy and a unit vector parallel to the magnetization, respectively, of the $i$ th layer. Since the tunneling barrier is not regarded as ferromagnetic $\Delta_{c}=0$. The magnetizations are both in-plane, and we take $\mathbf{n}_{l}=(1,0,0)$ and $\mathbf{n}_{r}=(\cos \phi, \sin \phi, 0)$, where $\phi$ is the angle between the magnetization vectors of the ferromagnetic electrodes. Finally, $\mathcal{I}$ and $\sigma=\left(\sigma_{x}, \sigma_{y}, \sigma_{z}\right)$ are the unit matrix in spinor space and the Pauli matrices, respectively.

Since the transverse modes $\mathbf{k}_{\|}$of the conduction electrons are conserved during the tunneling process, the calculation of the transmission probability reduces to a one-dimensional 
problem. The wave functions found when solving the resulting stationary Pauli-Schrödinger equation for a spin- $\sigma$ electron incoming from the left are [36]

$$
\begin{aligned}
& \psi_{l \sigma}(z)=\frac{1}{\sqrt{k_{\sigma}}} e^{i k_{\sigma} z} \chi_{l \sigma}+r_{\sigma, \sigma} e^{-i k_{\sigma} z} \chi_{l \sigma}+r_{\bar{\sigma}, \sigma} e^{-i k_{\bar{\sigma}} z} \chi_{l \bar{\sigma}} \\
& \psi_{c \sigma}(z)=\sum_{i= \pm}\left(C_{\sigma, i} e^{q z}+D_{\sigma, i} e^{-q z}\right) \chi_{l i} \\
& \psi_{r \sigma}(z)=t_{\sigma, \sigma} e^{i \kappa_{\sigma} z} \chi_{r \sigma}+t_{\bar{\sigma}, \sigma} e^{i \kappa_{\bar{\sigma}} z} \chi_{r \bar{\sigma}}
\end{aligned}
$$

where $k_{\sigma}=\sqrt{k_{\sigma 0}^{2}-k_{\|}^{2}}, q=\sqrt{q_{0}^{2}+k_{\|}^{2}}$, and $\kappa_{\sigma}=\sqrt{\kappa_{\sigma 0}^{2}-k_{\|}^{2}}$, with

$$
\begin{aligned}
k_{\sigma 0} & =\sqrt{\frac{2 m_{l}^{*}}{\hbar^{2}}\left(E+\sigma \frac{\Delta_{l}}{2}\right)}, \\
q_{0} & =\sqrt{\frac{2 m_{c}^{*}}{\hbar^{2}}\left(V_{c}+\mu_{0}-E\right)}, \\
\kappa_{\sigma 0} & =\sqrt{\frac{2 m_{r}^{*}}{\hbar^{2}}\left(E+\sigma \frac{\Delta_{r}}{2}\right)},
\end{aligned}
$$

and

$$
\chi_{l \sigma}=\frac{1}{\sqrt{2}}\left(\begin{array}{l}
1 \\
\sigma
\end{array}\right), \quad \chi_{r \sigma}=\frac{1}{\sqrt{2}}\left(\begin{array}{c}
1 \\
\sigma e^{i \phi}
\end{array}\right)
$$

where $\sigma=\uparrow(1), \downarrow(-1)$. The height of the barrier, $V_{c}$, is measured from the chemical potential, $\mu_{0}$, which at low temperatures is approximated by the Fermi energy. The coefficient $t_{\sigma, \sigma}\left(t_{\bar{\sigma}, \sigma}\right)$ represents the transmission probability amplitude for a tunneling process in which the electron spin is preserved (flipped). Similarly, $r_{\sigma, \sigma}$ and $r_{\bar{\sigma}, \sigma}$ are the reflection probability amplitudes. These amplitudes are computed analytically by solving the set of linear equations obtained when imposing the boundary conditions

$$
\begin{aligned}
\psi_{i \sigma}\left(z_{i c}\right) & =\psi_{c \sigma}\left(z_{i c}\right), \\
\left.\frac{1}{m_{i}^{*}} \frac{d \psi_{i \sigma}}{d z}\right|_{z=z_{i c}} & =\left.\frac{1}{m_{c}^{*}} \frac{d \psi_{c \sigma}}{d z}\right|_{z=z_{i c}},
\end{aligned}
$$

where $i=l, r$ and $z_{i c}$ is the position of the interface between the central and the $i$ th layer. The transmission probability is now computed through

$$
T_{\sigma}\left(E, \mathbf{k}_{\|}\right)=\frac{m_{l}^{*}}{m_{r}^{*}}\left(\kappa_{\sigma}\left|t_{\sigma, \sigma}\right|^{2}+\kappa_{\bar{\sigma}}\left|t_{\bar{\sigma}, \sigma}\right|^{2}\right) .
$$

The total transmission probability is $T=T_{\uparrow}+T_{\downarrow}$.

The computation of the transmission amplitudes is, in general, quite cumbersome. However, in the limit $q d \gg 1$, the following simplified analytical expression for the coefficients $t_{\sigma, \sigma}$ and $t_{\bar{\sigma}, \sigma}$ is found [36]:

$$
t_{\sigma, \sigma^{\prime}} \approx-\frac{2 i m_{c}^{*} m_{r}^{*} q \sqrt{k_{\sigma}}}{\left(m_{l}^{*} q-i m_{c}^{*} k_{\sigma}\right)\left(m_{r}^{*} q-i m_{c}^{*} \kappa_{\sigma^{\prime}}\right)}\left(1+\sigma \sigma^{\prime} e^{-i \phi}\right) e^{-q d},
$$

which is valid to first order in $\exp (-q d)$. Therefore, replacing Eq. (12) in Eq. (11) one obtains the transmission probability:

$$
\begin{aligned}
& T_{\sigma}\left(E, k_{\|}\right) \\
& \approx \frac{8 m_{l}^{*} m_{r}^{*} m_{c}^{* 2} k_{\sigma}\left(\kappa_{\sigma}+\kappa_{\bar{\sigma}}\right)\left(m_{r}^{* 2} q^{2}+m_{c}^{* 2} \kappa_{\sigma} \kappa_{\bar{\sigma}}\right) q^{2}}{\left(m_{l}^{* 2} q^{2}+m_{c}^{* 2} k_{\sigma}^{2}\right)\left(m_{r}^{* 2} q^{2}+m_{c}^{* 2} \kappa_{\sigma}^{2}\right)\left(m_{r}^{* 2} q^{2}+m_{c}^{* 2} \kappa_{\bar{\sigma}}^{2}\right)} \\
& \quad \times\left[1+\frac{\left(\kappa_{\sigma}-\kappa_{\bar{\sigma}}\right)\left(m_{r}^{* 2} q^{2}-m_{c}^{* 2} \kappa_{\sigma} \kappa_{\bar{\sigma}}\right)}{\left(\kappa_{\sigma}+\kappa_{\bar{\sigma}}\right)\left(m_{r}^{* 2} q^{2}+m_{c}^{* 2} \kappa_{\sigma} \kappa_{\bar{\sigma}}\right)} \cos \phi\right] e^{-2 q d} .
\end{aligned}
$$

Notice that $T\left(E, \mathbf{k}_{\|}\right)=T\left(E, k_{\|}\right)$.

\section{Analytical expression for the TMT}

To compute the thermopower [Eq. (4a)] and the conductance [Eq. (4b)], the transverse modes $k_{\|}$in Eq. (13) need to be integrated out [Eq. (3)], which can only be done numerically. However, for $k_{\|} \ll q_{0}$ the wave vector $q$ can be approximated as $q=q_{0}\left[1+\left(k_{\|} / \sqrt{2} q_{0}\right)^{2}\right]$. In such a limit, the $k_{\|}$dependence of the transmission probability [Eq. (13)] is dominated by the exponential factor $\exp \left(-k_{\|}^{2} d / q_{0}\right)$. Therefore, for relatively small $q_{0}$ and large barrier thicknesses, the transmission probability rapidly decay with increasing $k_{\|}$and the main contribution to the integral in Eq. (3) comes from the vicinity of $k_{\|} \approx 0$. Under this approximation Eq. (3) becomes

$$
g(E) \approx \frac{e^{2} q_{0}}{8 \pi^{2} \hbar d} T(E, 0) .
$$

Substituting explicitly the expression for $T(E, 0)$ [Eq. (13)] in Eq. (14) gives

$$
g(E) \approx g_{0}\left[1+P_{g l}^{\mathrm{eff}} P_{g r}^{\mathrm{eff}} \cos \phi\right],
$$

where $g_{0}=g_{l 0} g_{r 0}$, with

$$
\begin{aligned}
g_{l 0}= & \sqrt{\frac{2 e^{2} q_{0} e^{-2 q_{0} d}}{\pi h d}} \\
& \times\left[\frac{m_{l}^{*} m_{c}^{*}\left(k_{\uparrow 0}+k_{\downarrow 0}\right)\left(m_{l}^{* 2} q_{0}^{2}+m_{c}^{* 2} k_{\uparrow 0} k_{\downarrow 0}\right)}{\left(m_{l}^{* 2} q_{0}^{2}+m_{c}^{* 2} k_{\uparrow 0}^{2}\right)\left(m_{l}^{* 2} q_{0}^{2}+m_{c}^{* 2} k_{\downarrow 0}^{2}\right)}\right] q_{0},
\end{aligned}
$$

and

$$
P_{g l}^{\mathrm{eff}}=\frac{\left(k_{\uparrow 0}-k_{\downarrow 0}\right)}{\left(k_{\uparrow 0}+k_{\downarrow 0}\right)} \frac{\left(m_{l}^{* 2} q_{0}^{2}-m_{c}^{* 2} k_{\uparrow 0} k_{\downarrow 0}\right)}{\left(m_{l}^{* 2} q_{0}^{2}+m_{c}^{* 2} k_{\uparrow 0} k_{\downarrow 0}\right)}
$$

is the effective spin polarization of the left electrode. The expression for $g_{r 0}\left(P_{g r}^{\text {eff }}\right)$ is found by replacing in Eq. (16) [Eq. (17)] $k_{\sigma 0}$ and $m_{l}^{*}$ with $\kappa_{\sigma 0}$ and $m_{r}^{*}$, respectively.

In order now to derive analytical expressions for the thermopower and the TMT, we benefit from Mott's relation $[33,34]$, which states that

$$
S=-\left.\frac{\pi^{2}}{3} \frac{k_{B}^{2}}{e} \frac{d}{d E} \ln g(E)\right|_{E=\mu_{0}} T_{0},
$$

where $k_{B}$ is the Boltzmann constant. Equation (18) allows to compute the thermopower given the energy dependent conductance $g(E)$. Thus, replacing Eq. (15) into Eq. (18) yields 
the following expression for the thermopower:

$$
S(\phi) \approx\left[\frac{1+(1-\eta) P_{g l}^{\mathrm{eff}}\left(\mu_{0}\right) P_{g r}^{\mathrm{eff}}\left(\mu_{0}\right) \cos \phi}{1+P_{g l}^{\mathrm{eff}}\left(\mu_{0}\right) P_{g r}^{\mathrm{eff}}\left(\mu_{0}\right) \cos \phi}\right] S_{0},
$$

where

$$
\eta=\left.\frac{\pi^{2}}{3} \frac{k_{B}^{2}}{e} \frac{1}{S_{0}} \frac{d}{d E} \ln \left(P_{g l}^{\mathrm{eff}} P_{g r}^{\mathrm{eff}}\right)\right|_{E=\mu_{0}} T_{0},
$$

is a dimensionless quantity and $S_{0} \equiv S(\pi / 2)$. The explicit form for the parameter $\eta$ is given in Appendix A. Finally, the expression for the TMT is found by replacing Eq. (19) into Eq. (5), which reads

$$
\begin{aligned}
\operatorname{TMT}(\phi) \approx & -\frac{P_{g l}^{\mathrm{eff}}\left(\mu_{0}\right) P_{g r}^{\mathrm{eff}}\left(\mu_{0}\right)}{1+P_{g l}^{\mathrm{eff}}\left(\mu_{0}\right) P_{g r}^{\mathrm{eff}}\left(\mu_{0}\right)} \\
& \times \frac{\eta(1-\cos \phi)}{1+(1-\eta) P_{g l}^{\mathrm{eff}}\left(\mu_{0}\right) P_{g r}^{\text {eff }}\left(\mu_{0}\right) \cos \phi} .
\end{aligned}
$$

Equations (19) and (21) represent the thermal analogs of Slonczewski's formulas for the conductance and the TMR, respectively [32,36].

An interesting limit to study is that in which Julliere's model is valid $[36,38]$. In such case, the effective spin polarization reduces to $[36,38]$

$$
P_{g i}^{\mathrm{eff}}=\frac{D_{i}^{\uparrow}-D_{i}^{\downarrow}}{D_{i}^{\uparrow}+D_{i}^{\downarrow}}, \quad i=l, r,
$$

where $D_{i}^{\sigma}$ is the spin-dependent density of states in the $i$ th electrode. Moreover, since $2 g_{0}=g(\phi=0)+g(\phi=\pi)$ [see Eq. (15)], then [36]

$$
g_{0} \propto \frac{1}{2}\left(D_{l}^{\uparrow} D_{r}^{\uparrow}+D_{l}^{\downarrow} D_{r}^{\downarrow}+D_{l}^{\uparrow} D_{r}^{\downarrow}+D_{l}^{\downarrow} D_{r}^{\uparrow}\right),
$$

which allows us also to compute $S_{0}$ from the density of states and, hence, the $\eta$ parameter [Eq. (20)]. Therefore, in the limit where Julliere's model and Mott's law are valid, one can estimate both the thermopower [Eq. (19)] and the TMT [Eq. (21)] only through the density of states.

\section{RESULTS}

In this section we discuss the results obtained for the case of an $\mathrm{Fe} / \mathrm{Al}_{2} \mathrm{O}_{3} / \mathrm{Fe}$ MTJ. Since the system is symmetric, the effective spin polarization [Eq. (17)] is the same in both electrodes, so $P_{g l}^{\mathrm{eff}}=P_{g r}^{\mathrm{eff}} \equiv P$. In addition, for the set of parameters discussed throughout this section, the relation $P^{2} \ll 1$ is satisfied, hence, Eq. (19) takes the simple form

$$
S(\phi) \approx\left[1-\eta P^{2} \cos \phi\right] S_{0} .
$$

This cosine-like behavior found for the angular dependence of the thermopower has been observed in Refs. $[15,16]$, where they measure the induced thermovoltage for $\mathrm{CoFeB} / \mathrm{MgO} / \mathrm{CoFeB}$ MTJs. However, ab initio calculations performed in Ref. [21] for the same kind of MTJs show a different behavior. This might be an indication that for the structure considered in Ref. [21] the condition $P^{2} \ll 1$ is not fulfilled. In such a case terms of higher order in $\cos \phi$ which are present in the expansion of $S$ [Eq. (19)] become relevant and deviations from the angular dependence given in
Eq. (24) occur. Furthermore, replacing Eq. (24) in the general expression for the TMT [Eq. (5)] yields

$$
\operatorname{TMT}(\phi) \approx-\eta P^{2}(1-\cos \phi),
$$

where we have used that $\eta P^{2} \ll 1$.

The thermopower and TMT coefficients can be evaluated at different levels of approximation. The simplest one is to use the analytical expressions obtained in the previous section, namely, Eqs. (19) and (21). These analytical expressions combine both Slonczewski's and Mott's models. We therefore refer to them as the Slonczewski-Mott approximation. A step forward consists of disregarding Mott's approximation, i.e., to use the analytical expression for $g$ [Eq. (14)] but to compute numerically the energy integrations in Eqs. (4a) and (4b). This is what we call the Slonczewski approximation. Finally, exact results are obtained by disregarding both Slonczewski's and Mott's approximations; i.e., by performing numerical integration in Eqs. (3), (4a), and (4b).

For quantitative illustration we considered a symmetric MTJ with $k_{\sigma 0}=\kappa_{\sigma_{0}}$ and the following model parameters: $m_{l}^{*}=m_{r}^{*}=m_{0}, m_{c}^{*}=0.4 m_{0}$ (here $m_{0}$ is the bare electron mass), $k_{\uparrow 0}\left(\mu_{0}\right)=1.09 \AA^{-1}$, and $k_{\downarrow 0}\left(\mu_{0}\right)=0.42 \AA^{-1}$.

For the low temperature considered here $(T=4.2 \mathrm{~K})$ we found that the agreement between both the Slonczewski-Mott and Slonczewski approximations was extremely good. The calculations corresponding to the Slonczewski approximation are therefore omitted in all the figures.

Figure 2 shows the TMT dependence on the relative magnetization $\phi$ for different values of the barrier height $V_{c}$. The agreement between the exact calculations (solid lines) and Slonczewski-Mott approximation (dashed lines) is reasonably good already for a $40 \AA$ thick barrier and the observed angular dependence is well described by Eq. (25). Notice how the sign of the TMT changes as the value of $V_{c}$ increases. Let us now discuss more in detail this sign change.

Figures 3(a) and 3(b) show the dependence of the exact and approximate thermopowers, respectively, on the barrier height. Dashed lines correspond to the case when the magnetizations are parallel $(\phi=0)$ and solid lines to the antiparallel

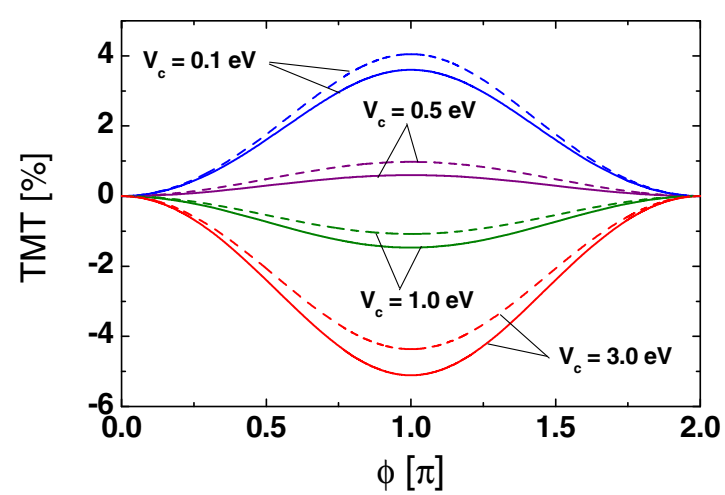

FIG. 2. (Color online) TMT dependence of an $\mathrm{Fe} / \mathrm{Al}_{2} \mathrm{O}_{3} / \mathrm{Fe}$ MTJ on the relative orientation of the magnetizations of the ferromagnets, for different values of the tunneling barrier height $V_{c}$, and thickness $d=40 \AA$. Solid lines represent the exact calculations while dashed lines correspond to the Slonczewski-Mott approximation. 


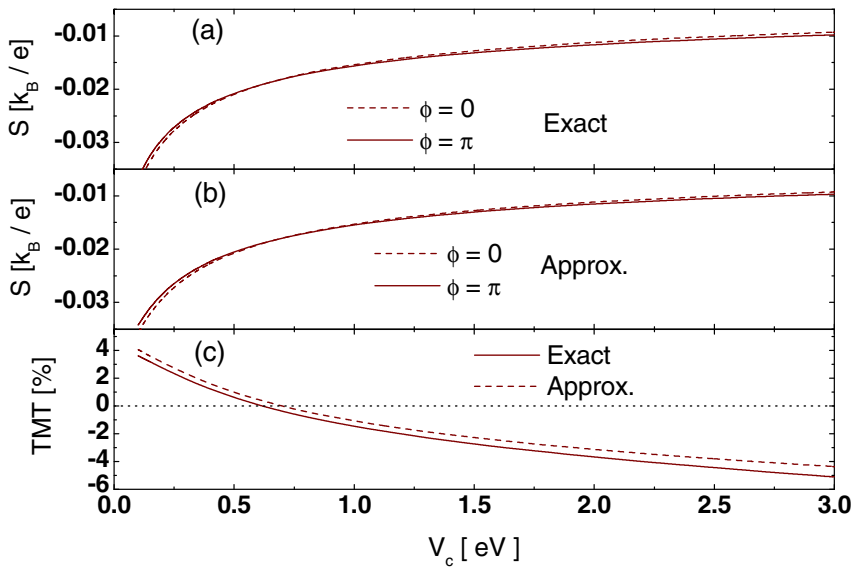

FIG. 3. (Color online) (a) Thermopower of an $\mathrm{Fe} / \mathrm{Al}_{2} \mathrm{O}_{3} / \mathrm{Fe}$ MTJ as a function of the barrier height. The solid (dashed) line corresponds to the exact calculation for the antiparallel (parallel) configuration. (b) Same as (a) but within the Slonczewski-Mott approximation. (c) $\operatorname{TMT}(\pi)$ dependence on the barrier height. Solid and dashed lines correspond, respectively, to the exact and approximated results. Notice that the thermopowers for the parallel and antiparallel orientations cross, meaning the TMT becomes zero. The barrier thickness is $d=40 \AA$.

magnetizations $(\phi=\pi)$. Figure 3(c) shows the TMT dependence on the barrier height when $\phi=\pi$. Again, the agreement between the exact calculations (solid line) and the Slonczewski-Mott approximation (dashed line) is very good and it gets better at smaller values of $V_{c}$. A similar behavior was obtained for the case of the TMR [39].

The TMT is a complicated function of $V_{c}$. Therefore, the trend observed in Fig. 3(c) is not as straightforward to understand as the angular dependence displayed in Fig. 2. In fact, Fig. 3(c) shows that the TMT changes sign for a specific value of the barrier height $V_{c}^{(0)}$, which corresponds to the crossing between the thermopower for the parallel and the antiparallel cases [see Figs. 3(a) and 3(b)]. The condition for $\operatorname{TMT}(\phi)=0$ [Eq. (21)] is satisfied when $P_{g i}^{\text {eff }}\left(\mu_{0}\right)=0$, which according to Eq. (17) occurs when

$$
V_{c}^{(0)}=\frac{\hbar^{2} k_{\mu \uparrow} k_{\mu \downarrow}}{2\left(m_{0}^{2} / m_{c}^{*}\right)},
$$

where $k_{\mu \uparrow}=k_{\uparrow 0}\left(\mu_{0}\right)$ and $k_{\mu \downarrow}=k_{\downarrow 0}\left(\mu_{0}\right)$. For this same value the TMR also becomes zero [36], although it remains positive. The sign change of the TMT is not related to the effective spin polarization but to a sign change in the parameter $\eta$. This is particularly clear for the case of a symmetric MTJ, since for symmetric MTJs the TMT is proportional to $\eta P^{2}$ [Eq. (25)].

Figures 4(a) and 4(b) show the thermopower dependence on the barrier thickness $d$ when the magnetizations are antiparallel $(\phi=\pi)$. The barrier thickness only enters in the denominator of $\eta$ (see Appendix A). Therefore, as $d$ increases $\eta \rightarrow 0$ and, hence, $S \rightarrow S_{0}$ [Eq. (19)]. Furthermore, according to Eq. (A2), $S_{0}$ depends linearly on $d$. This explains the linear behavior of $S$ displayed in Figs. 4(a) and 4(b).

The dependence of the TMT coefficient in the antiparallel configuration $(\phi=\pi)$ is shown in Fig. 4(c) for two different

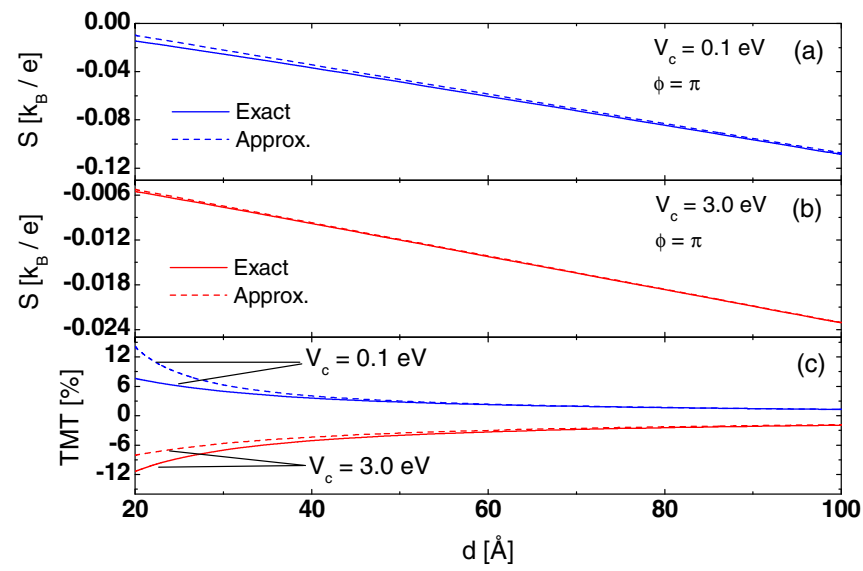

FIG. 4. (Color online) (a) Thermopower of an $\mathrm{Fe} / \mathrm{Al}_{2} \mathrm{O}_{3} / \mathrm{Fe}$ MTJ as a function of the barrier thickness for the antiparallel configuration $(\phi=\pi)$ and the barrier hight $V_{c}=0.1 \mathrm{eV}$. (b) Same as (a) but for $V_{c}=3.0 \mathrm{eV}$. (c) $\mathrm{TMT}(\pi)$ dependence on the barrier thickness. In (a), (b), and (c) the solid lines represent the exact calculations and the dashed lines correspond to the Slonczewski-Mott approximation.

values of the barrier hight. As discussed above, $\eta$ approaches zero as $d$ increases. Since the TMT is proportional to $\eta$ [see Eq. (21)], the TMT is suppressed for large barrier thicknesses, as seen in Fig. 4(c). This differs from the thermopower, whose amplitude increases with $d$ [see Figs. 4(a) and 4(b)]. This behavior agrees with the low temperature trend found in the ab initio calculations in Ref. [26] for a $\mathrm{CoFeB} / \mathrm{MgO} / \mathrm{CoFeB}$ MTJ, where the dependence on the thickness of the barrier was studied by varying the number of $\mathrm{MgO}$ monolayers.

By comparing the exact (solid lines) and approximate (dashed lines) results shown in Fig. 4, one notices that the Slonczewski-Mott approximation works better for thicker barriers. This is a typical feature of the Slonczewski approximation [Eq. (14)] which has also been observed in theoretical investigations of the TMR [39]. It is intrinsic to the derivation of Eq. (14), which is only valid for large values of $d$.

Finally, for a symmetric MTJ the effective spin polarization $P$ can be extracted from TMR experiments, by measuring the parallel and antiparallel conductances $G_{P}$ and $G_{\mathrm{AP}}$, respectively, through the equation [36]

$$
P= \pm \sqrt{\frac{G_{P}-G_{\mathrm{AP}}}{G_{P}+G_{\mathrm{AP}}}} .
$$

Therefore, by measuring the parallel, $S_{P}$, and antiparallel, $S_{\mathrm{AP}}$, values of the thermopower, it is possible to experimentally estimate the parameter $\eta$ with the equation

$$
\eta=-\frac{2 G_{P} G_{\mathrm{AP}}}{G_{P} S_{P}+G_{\mathrm{AP}} S_{\mathrm{AP}}} \frac{S_{P}-S_{\mathrm{AP}}}{G_{P}-G_{\mathrm{AP}}},
$$

found when replacing Eq. (27) in Eq. (21).

\section{SUMMARY}

We have studied thermal spin transport in magnetic tunnel junctions using the thermal analog of Slonczewski's model of 
tunneling. We have derived analytical expressions for the thermopower and the tunneling magnetothermopower of magnetic junctions in both Slonczewski's approximation and in the limit of Julliere's model. We show that the TMT can be both positive and negative, depending on the barrier properties, crossing through zero at a certain barrier height. Our expressions could be used in combination with first-principles band structure parameters of the bulk ferromagnetic materials forming the junction to estimate the spin thermal transport characteristics.
Furthermore, they can serve as a phenomenological description of experiments on junction magnetothermopower, in most cases with a single fitting parameter $(\eta)$.

\section{ACKNOWLEDGMENT}

The authors are grateful for the financial support offered by the Deutsche Forshungsgemeinschaft (DFG) via the Priority Program "Spin Caloric Transport" (SPP 1538).

\section{APPENDIX A: THE $\eta$ PARAMETER}

The expression found for $\eta$ is

$$
\eta=-\frac{\pi^{2}}{3} \frac{k_{B}^{2}}{e} \frac{T_{0}}{S_{0}} \sum_{i=l, r} \frac{2 m_{i}^{*}}{\hbar^{2} k_{i \uparrow} k_{i \downarrow}}\left(1+\frac{m_{i}^{* 2} m_{c}^{* 2} q_{F}^{2}\left(k_{i \downarrow}^{2}+k_{i \uparrow}^{2}\right)+2 m_{i}^{*} m_{c}^{* 3} k_{i \uparrow}^{2} k_{i \downarrow}^{2}}{m_{i}^{* 4} q_{F}^{4}-m_{c}^{* 4} k_{i \uparrow}^{2} k_{i \downarrow}^{2}}\right),
$$

where

$$
\begin{aligned}
S_{0}= & -\frac{\pi^{2}}{3} \frac{k_{B}^{2}}{e} T_{0}\left\{\frac{2 m_{c}^{*}}{\hbar^{2} q_{F}^{2}}\left(q_{F} d-\frac{3}{2}\right)+\frac{1}{\hbar^{2}} \sum_{i=l, r}\left[\frac{m_{i}^{*}}{k_{i \uparrow} k_{i \downarrow}}\left(1+\frac{m_{c}^{* 2}\left(k_{i \uparrow}^{2}+k_{i \downarrow}^{2}\right)-2 m_{i}^{*} m_{c}^{*} k_{i \uparrow} k_{i \downarrow}}{m_{i}^{* 2} q_{F}^{2}+m_{c}^{* 2} k_{i \uparrow} k_{i \downarrow}}\right)\right.\right. \\
& \left.\left.-\left(\frac{2 m_{i}^{* 2} q_{F}^{2}+m_{c}^{* 2}\left(k_{i \uparrow}^{2}+k_{i \downarrow}^{2}\right)}{\left(m_{i}^{* 2} q_{F}^{2}+m_{c}^{* 2} k_{i \uparrow}^{2}\right)\left(m_{i}^{* 2} q_{F}^{2}+m_{c}^{* 2} k_{i \downarrow}^{2}\right)}\right) 2 m_{i}^{*} m_{c}^{*}\left(m_{c}^{*}-m_{i}^{*}\right)\right]\right\},
\end{aligned}
$$

and $k_{l \sigma}=k_{\sigma 0}\left(\mu_{0}\right), q_{F}=q_{0}\left(\mu_{0}\right)$, and $k_{r \sigma}=\kappa_{\sigma 0}\left(\mu_{0}\right)$. Since the effective masses and Fermi wave vectors can be computed through ab initio calculations, it is also possible to estimate the parameter $\eta$.

[1] I. Žutić, J. Fabian, and S. Das Sarma, Rev. Mod. Phys. 76, 323 (2004).

[2] G. E. W. Bauer, E. Saitoh, and B. J. van Wees, Nat. Mater. 11, 391 (2012).

[3] R. Barnard, Thermoelectricity in Metals and Alloys (Taylor \& Francis, London, 1972).

[4] M. Johnson and R. H. Silsbee, Phys. Rev. B 35, 4959 (1987).

[5] M. Johnson, J. Supercond. 16, 679 (2003).

[6] L. Gravier, S. Serrano-Guisan, F. Reuse, and J.-P. Ansermet, Phys. Rev. B 73, 024419 (2006).

[7] L. Gravier, S. Serrano-Guisan, F. Reuse, and J.-P. Ansermet, Phys. Rev. B 73, 052410 (2006).

[8] K. Uchida, S. Takahashi, K. Harii, J. Ieda, W. Koshibae, K. Ando, S. Maekawa, and E. Saitoh, Nature (London) 455, 778 (2008).

[9] A. Slachter, F. L. Bakker, J.-P. Adam, and B. J. van Wees, Nat. Phys. 6, 879 (2010).

[10] K. Uchida, J. Xiao, H. Adachi, J. Ohe, S. Takahashi, J. Ieda, T. Ota, Y. Kajiwara, H. Umezawa, H. Kawai, G. E. W. Bauer, S. Maekawa, and E. Saitoh, Nat. Mater. 9, 894 (2010).

[11] C. M. Jaworski, J. Yang, S. Mack, D. D. Awschalom, J. P. Heremans, and R. C. Myers, Nat. Mater. 9, 898 (2010).

[12] D. Qu, S. Y. Huang, J. Hu, R. Wu, and C. L. Chien, Phys. Rev. Lett. 110, 067206 (2013).

[13] J.-C. Le Breton, S. Sharma, H. Saito, S. Yuasa, and R. Jansen, Nature (London) 475, 82 (2011).

[14] M. Walter, J. Walowski, V. Zbarsky, M. Mnzenberg, M. Schfers, D. Ebke, G. Reiss, A. Thomas, P. Peretzki, M. Seibt, J. S.
Moodera, M. Czerner, M. Bachmann, and C. Heiliger, Nat. Mater. 10, 742 (2011).

[15] N. Liebing, S. Serrano-Guisan, K. Rott, G. Reiss, J. Langer, B. Ocker, and H. W. Schumacher, Phys. Rev. Lett. 107, 177201 (2011).

[16] Z. H. Zhang, Y. S. Gui, L. Fu, X. L. Fan, J. W. Cao, D. S. Xue, P. P. Freitas, D. Houssameddine, S. Hemour, K. Wu, and C.-M. Hu, Phys. Rev. Lett. 109, 037206 (2012).

[17] W. Lin, M. Hehn, L. Chaput, B. Negulescu, S. Andrieu, F. Montaigne, and S. Mangin, Nat. Commun. 3, 744 (2012).

[18] F. K. Dejene, J. Flipse, and B. J. van Wees, Phys. Rev. B 86, 024436 (2012).

[19] Z.-C. Wang, G. Su, and S. Gao, Phys. Rev. B 63, 224419 (2001).

[20] E. McCann and V. I. Fal'ko, Phys. Rev. B 66, 134424 (2002).

[21] M. Czerner, M. Bachmann, and C. Heiliger, Phys. Rev. B 83, 132405 (2011).

[22] R. Jansen, A. M. Deac, H. Saito, and S. Yuasa, Phys. Rev. B 85, 094401 (2012).

[23] M. Hatami, G. E. W. Bauer, Q. Zhang, and P. J. Kelly, Phys. Rev. B 79, 174426 (2009).

[24] B. Scharf, A. Matos-Abiague, I. Žutić, and J. Fabian, Phys. Rev. B 85, 085208 (2012).

[25] N. Liebing, S. Serrano-Guisan, P. Krzysteczko, K. Rott, G. Reiss, J. Langer, B. Ocker, and H. W. Schumacher, Appl. Phys. Lett. 102, 242413 (2013).

[26] M. Czerner and C. Heiliger, J. Appl. Phys. 111, 07C511 (2012).

[27] C. Heiliger, C. Franz, and M. Czerner, Phys. Rev. B 87, 224412 (2013). 
[28] X. Jia and K. Xia, AIP Advances 2, 041411 (2012).

[29] I. J. Vera-Marun, B. J. van Wees, and R. Jansen, Phys. Rev. Lett. 112, 056602 (2014).

[30] W. Chen, Z. Zhang, L. Jiang, Z. Xing, R. Shen, and B. Wang, Eur. Phys. J. B 85, 1 (2012).

[31] Y. Takezoe, K. Hosono, A. Takeuchi, and G. Tatara, Phys. Rev. B 82, 094451 (2010).

[32] J. C. Slonczewski, Phys. Rev. B 39, 6995 (1989).

[33] N. Mott and H. Jones, The Theory of the Properties of Metals and Alloys (Dover, Mineola, NY, 1958).
[34] M. Cutler and N. F. Mott, Phys. Rev. 181, 1336 (1969).

[35] H. Callen, Thermodynamics and an Introduction to Thermostatistics (Wiley, New York, 1985).

[36] J. Fabian, A. Matos-Abiague, C. Ertler, P. Stano, and I. Žutić, Acta. Phys. Slov. 57, 565 (2007)

[37] E. C. Stoner, Proc R. Soc. London A 165, 372 (1938).

[38] M. Julliere, Phys. Lett. A 54, 225 (1975).

[39] J. M. MacLaren, X.-G. Zhang, and W. H. Butler, Phys. Rev. B 56, 11827 (1997). 